$\mathrm{J}$ o u r n a l of

Mathematics

and Applications

JMA No 41, pp 63-79 (2018)

\title{
On Population Dynamics with Campaign on Contraception as Control Strategy
}

\author{
Virtue U. Ekhosuehi and Fidelis O. Chete
}

\begin{abstract}
This work considers a population divided into two groups according to the adoption of contraception. The campaign in favour of contraception is modelled as a bounded optimal control problem within the framework of the logistic and the Malthusian models of population dynamics. The control is the fraction of non-adopters successfully educated on contraception. The objective is to maximise the number of non-adopters successfully educated on contraception over time. The optimisation problem is solved using the Pontryagin's maximum principle and the parameters of the model are estimated using the method of least squares.
\end{abstract}

AMS Subject Classification: 49J15, 92D25.

Keywords and Phrases: Contraception; Logistic model; Malthusian model; Optimal control model; Population.

\section{Introduction}

The subject of population expansion and control has received considerable attention in the literature (for instance, $[23,36]$ ). The need to control the rate of population expansion has led to the introduction of several programmes on the use of contraceptives in many developing countries [21]. For example, in Nigeria (with which the authors are acquainted), the 'Get it together' campaign has been introduced to sensitise the masses on the use of contraceptives. The commonly used contraceptives include condom, diaphragm, vaginal cream/foaming tablets, oral contraceptives or pills, Intra-Uterine Device (IUD), implant and sterilization. These contraceptives are 
accepted globally for birth control $[9,21]$. There is no need to stress the different contraceptives and how it has been accepted worldwide as this can be found elsewhere $[9,26,35,36]$. The practice of birth control in Japan, Russia, Puerto Rico, China, India and Cameroon has been reported in the literature [26]. The campaign on birth control is usually inexpensive for the social crusaders ${ }^{1}$ (that is, the birth control advocates). This is because fertility control are subsidized in both high- and low-income countries [28]. On the whole condoms are distributed inexpensively and IUD insertion costs little [8]. This ought to be enough motivation for many adults to adopt the use of contraceptives. Regardless of the campaign to create awareness on use of contraceptives some individuals still hold on to their belief and may get involved in unprotected sex so much so that it may result to unplanned births, sexually transmitted diseases and child abandonment. Early research has shown that factors such as fear of the unknown effects of contraceptives, spouse's disapproval, religious and cultural beliefs, inadequate information and poor service of family planning clinics, may be barriers to use of contraceptives [21]. The difficulty in getting the population to accept the use of contraceptives is a problem, particularly in rural areas of developing countries. This problem is the motive for the continuous research on awareness creation on birth control with the use of contraceptives [21].

This paper considers a system made up of individuals that have attained the reproductive (or child-bearing) age and focuses on the use of a method of contraception (e.g., condoms). The study is aimed at deriving the optimal number of non-adopters that should be successfully educated on contraception using optimal control theory. Models based on optimal control theory are well-known in the literature $[1,5,6]$. The increased application of optimal control theory in ecology and natural resource management has been discussed as well [29]. In this present study, the state variables, which are the adopters and the non-adopters of the use of contraceptives, coexist. The control variable is the fraction of non-adopters successfully educated on contraception (i.e., the new adopters). This control is used as a proxy for the campaign effort. The use of a fraction of the population as a control is not novel as it has earlier been considered [23]. Before delving into the mathematical formulation of the population dynamics, we provide a review on methods of birth control and population models in Section 2. The model formulation is given in Section 3. Section 4 is concerned with a numerical illustration of the population dynamics, and Section 5 concludes the paper.

\section{Related Works}

Birth control is crucial to reducing population expansion [23] and poverty [36] in developing economies. The gains of birth control, inter alia, include: a smaller population, higher Gross National Product (GNP) per head and reduction in the ratio of dependent children to work-age population [8]. The methods of birth control are found in the literature [21, 32]. These include: the long-term methods such as IntraUterine Device (IUD), sterilization for both male and female and implant; the hor-

\footnotetext{
${ }^{1}$ The crusaders in a developing country like Nigeria are mainly physicians, social workers and non-governmental organisations.
} 
monal methods such as oral contraceptives, patch and ring; and the barrier methods such as the condoms, diaphragm and spermicides. It has been found that men who tend to assign contraceptive responsibility to women have more negative attitudes towards male contraceptive use [35]. The use of contraceptives among women and the factors that influence their use have been examined [4]. The factors include: being in a relationship, number of sex partners, pregnancy status, sexual activity status, age and social class. The prevalence of contraceptive use among women of reproductive age in Calabar, Nigeria has earlier been studied [7]. Lack of information as one of the factors that hinders the use of modern methods of birth control has been identified in Nigeria [21]. Essentially birth control is important in order to attain a steady-state growth rate of the population [23].

Population studies have gained prominence in the literature. Some of the early works on population dynamics are found in [16, 24]. Research on the relationship between population and economic growth has also been carried out [3]. In the study of pre-industrial societies, the Malthusian model of population dynamics occupies a central position in the analysis of the demographic change [2, 14, 19, 27]. A competing model of population dynamics to the Malthusian model is the logistic model. The Malthusian model is well-suited for populations that are not limited by space, while the logistic model is the standard model for single-species population growth [34]. The logistic equation, wherein the instantaneous birth rate per individual and the carrying capacity of the system are the parameters, is a more realistic model in terms of the birth and death processes of population growth [10]. The logistic curve provides reliable projections of the total population provided that there is a relationship among births, deaths and migration [13]. The work of [13] has been generalized [22]. It has been found that the population size of the logistic model with varying carrying capacity will eventually be gamma-distributed [25] and that population densities may exhibit oscillatory behaviour owing to seasonality [15].

Solutions to population models can be either exact or numerical. In [18] exact solutions to a quasi-linear first-order differential equation that models the growth of a single population subject to the logistic growth was found. However, in [11], numerical solutions based on the central finite difference method to the first-order hyperbolic equation of age and time variables which describes the one-sex models of population dynamics was provided. The existence of equilibrium solutions of a nonlinear structured population model and the local asymptotic stability of the equilibria has been proved [33].

Demographic and environmental variability and the possibility of extinction of a population may be modelled correctly in stochastic population models [37]. The structure variables include chronological age of each individual and the population size. In the stochastic population model it is possible to approximate the model as a diffusion [12]. In this case the population is at risk of extinction and the stochastic nature is caused by demographic and environmental fluctuations. The distribution of the extinction times in the stochastic logistic population model wherein the lifespan of any population can be described has been investigated [20]. In another study an alternative approach to the forecasting ability of the logistic population model was illustrated by modifying the assumption of the homoscedasticity of the error term 
[17]. Later on, a method that can be used to fit a population model in the presence of observation error was described [31]. This study improves on the existing population models in the literature $[2,10,14,19,27]$ by integrating the population dynamics and the effect of birth control campaign in the same dynamical system. The method of estimation of parameters for the state-transition equations is similar to the one found in $[31]$.

\section{Model Formulation}

In this section, we complete the statement of the problem alongside with the underlying assumptions and provide the solution.

\subsection{Model Development}

Consider a system which consists of individuals of reproductive (or child-bearing) age. Individuals in the system are assumed to be divided into two mutually exclusive compartments: non-adopters $(x(t))$ and adopters $(y(t))$. We assume that the babies and the people in child-bearing age are distinguishable. This assumption is necessary because the transition from non-adopter to adopter is only applicable to people in child-bearing age. Only a portion of $x(t)$ can transfer to adopters and the new-born babies cannot be adopter or non-adopter in less than a legally allowable child-bearing age (say, 15 years). We assume that the adopters and the non-adopters coexist in the system and their interaction precludes personal issues such as the use of contraceptives. This assumption is consistent with the setting in rural communities in developing countries like Nigeria, where sex education is seen as either a taboo or immoral [21]. The non-adopters may change their opinion due to a re-orientation campaign on the use of contraceptives provided by birth control crusaders (e.g. physicians, social workers and non-governmental organisations) by whatever means. We assume that the cost of the campaign, which includes the cost of consultation with physicians and social workers on the use of contraceptives, is negligible. This is because fertility control is subsidized in both high- and low-income countries [8, 28]. As a result cost is not considered within the model formulation.

Let $\theta(t)$ be the fraction of 'non-adopters successfully educated on contraception' (new adopters hereinafter). Then $\theta(t) x(t)$ is the number of new adopters attributed to the re-orientation campaign on the use of contraceptives. The changes in the total population are induced by two effects: maturity (the attainability of reproductive age) and attrition. On attaining the reproductive age, the new member of the system may be either adopter or non-adopter. The loss in population may be attributed to attrition such as death, emigration, or attaining menopause. It is reasonable to assume that the population dynamics of the birth-control adopters and non-adopters are different. Consequent upon this, the dynamics of the system is assumed to follow the population growth models below

$$
\frac{d x(t)}{d t}=\gamma_{1} x(t)-\gamma_{2} x^{2}(t)-\theta(t) x(t), x(0)=x_{0}
$$


and

$$
\frac{d y(t)}{d t}=\beta_{1} y(t)+\theta(t) x(t), y(0)=y_{0},
$$

where $\gamma_{1}$ is the intrinsic growth rate of non-adopters and $\beta_{1}$ is the intrinsic growth rate of adopters. The term $\gamma_{2} x^{2}(t)$ in equation (3.1) is used to model the loss of non-adopters induced by the non-use of contraceptives. This is realistic because ill health, which is one of the consequences of increased family size [9], is worse-off for the poorest (most of whom are in rural areas) that are most ignorant and apathetic on use of contraceptives [8]. The effect of interaction between the adopters and the non-adopters is not considered. Equations (3.1) and (3.2) are analogous to the wellknown logistic model and Malthusian model of population dynamics, respectively. In practice, equations (3.1) and (3.2) may be subjected to statistical analysis to ascertain their significance as an appropriate model for the two population compartments. We assume that the non-adopters may not all accept and practise contraception no matter the campaign owing to their religious beliefs. For this reason, the control $\theta(t)$ is assumed to satisfy the relation, $0 \leq \theta(t)<1$.

Let $\{t: 0<t \leq T\}$ be a fixed time horizon. Since efforts are made to increase the number of adopters, we define the objective function to be

$$
\max _{\theta(t)} \int_{0}^{T} \theta(t) x(t) d t .
$$

The optimal control problem posed by the objective function (3.3) and the state transition equations (3.1) and (3.2) together with the initial conditions and the bounds for the control is thus:

$$
\max _{\theta(t)} \int_{0}^{T} \theta(t) x(t) d t
$$

subject to

$$
\begin{gathered}
\frac{d x(t)}{d t}=\gamma_{1} x(t)-\gamma_{2} x^{2}(t)-\theta(t) x(t), \\
\frac{d y(t)}{d t}=\beta_{1} y(t)+\theta(t) x(t), \\
x(0)=x_{0}, y(0)=y_{0}, 0 \leq \theta(t)<1, t \in(0, T] .
\end{gathered}
$$

This model set-up is a bounded optimal control problem with the bounds being the closed-open interval $0 \leq \theta(t)<1$.

\subsection{Model Solution}

To solve the bounded control problem, we employ the Pontryagin's maximum principle. The analysis of our solution is as follows.

We compute the control function, $\theta(t)$, by assuming that its value is at the lower bound or it is in the interior. Suppose the total population is a variable, then the 
Hamiltonian, $H$, with arguments given as $\left(x(t), y(t), \theta(t), \lambda_{1}(t), \lambda_{2}(t)\right)$, for the problem is

$$
H=\theta(t) x(t)+\lambda_{1}(t)\left(\gamma_{1} x(t)-\gamma_{2} x^{2}(t)-\theta(t) x(t)\right)+\lambda_{2}(t)\left(\beta_{1} y(t)+\theta(t) x(t)\right),
$$

where $\lambda_{j}(t), j=1,2$, is a multiplier function, which defines the marginal valuation of the productive capacity of the respective state variables. The influence equations for the state variables $x(t)$ and $y(t)$ are obtained as

$$
\frac{d \lambda_{1}(t)}{d t}=-\frac{\partial H}{\partial x(t)}=-\left(\theta(t)+\lambda_{1}(t)\left(\gamma_{1}-2 \gamma_{2} x(t)-\theta(t)\right)+\lambda_{2}(t) \theta(t)\right)
$$

and

$$
\frac{d \lambda_{2}(t)}{d t}=-\frac{\partial H}{\partial y(t)}=-\beta_{1} \lambda_{2}(t)
$$

Thus,

$$
\lambda_{2}(t)=\varphi \exp \left(-\beta_{1} t\right),
$$

where $\varphi$ is a constant. Equation (3.7) implies that the marginal value of the adopters decays exponentially with time. The Lagrangian function, $L$, for the Hamiltonian subject to the control bounds, $0 \leq \theta(t)<1$, is

$$
\begin{aligned}
& L=\theta(t) x(t)+\lambda_{1}(t)\left(\gamma_{1} x(t)-\gamma_{2} x^{2}(t)-\theta(t) x(t)\right)+ \\
& \lambda_{2}(t)\left(\beta_{1} y(t)+\theta(t) x(t)\right)+\rho_{1} \theta(t)+\rho_{2}(1-\theta(t)),
\end{aligned}
$$

where $\rho_{1}$ and $\rho_{2}$ are the Lagrangian multipliers when the total population is a variable. The necessary conditions for $\theta(t)$ to maximise the bounded control problem are

$$
\frac{\partial L}{\partial \theta(t)}=x(t)-\lambda_{1}(t) x(t)+\lambda_{2}(t) x(t)+\rho_{1}-\rho_{2}=0, \rho_{1} \geq 0, \rho_{1} \theta(t)=0, \rho_{2}=0 .
$$

Without campaign on contraception, that is, $\theta(t)=0$, we use equation (3.9) to get

$$
\frac{d \lambda_{1}(t)}{d t} \geq \frac{d \lambda_{2}(t)}{d t}
$$

In this case, the optimal population, $x^{*}(t)+y^{*}(t)$, is obtained from the transition equations (3.1) and (3.2) by setting $\theta(t)=0$. Thus the optimal sub-populations are found by solving the respective state transition equations. We obtain

$$
x^{*}(t)=\frac{x_{0} \exp \left(\gamma_{1} t\right)}{\left(1-\frac{\gamma_{2}}{\gamma_{1}} x_{0}\left(1-\exp \left(\gamma_{1} t\right)\right)\right)},
$$

and

$$
y^{*}(t)=y_{0} \exp \left(\beta_{1} t\right)
$$

We use the symbol * to denote the optimal value. To increase the adopters, there is a need for awareness campaign. Such a campaign is effective when at least one 
non-adopter accepts and practises contraception. With campaign on contraception $\theta(t)$ lies in the open interval $(0,1)$. In this open interval, we obtain from equation (3.9) that

$$
\frac{d \lambda_{1}(t)}{d t}=\frac{d \lambda_{2}(t)}{d t}
$$

The optimal sub-populations, $x^{*}(t)$ and $y^{*}(t)$, are obtained using equation (3.7) to simplify the influence equation (3.5) and then the solution is substituted into the transition equations (3.1) and (3.2). We therefore obtain

$$
\begin{gathered}
x^{*}(t)=\frac{\gamma_{1}}{2 \gamma_{2}}-\frac{\beta_{1} \varphi \exp \left(-\beta_{1} t\right)}{2 \gamma_{2}\left(1+\varphi \exp \left(-\beta_{1} t\right)\right)} \\
y^{*}(t)=\exp \left(\beta_{1} t\right)\left(y_{0}+\vartheta_{1}\left(1-\exp \left(-\beta_{1} t\right)\right)+\vartheta_{2}\left(\ln (\zeta(t))^{2}-\wp(t)\right)\right)
\end{gathered}
$$

and

$$
\theta^{*}(t)=\gamma_{1}-\gamma_{2} x^{*}(t)-\frac{x^{\prime}(t)}{x^{*}(t)}
$$

where

$$
\begin{gathered}
\varphi=\frac{\gamma_{1}-2 \gamma_{2} x_{0}}{\beta_{1}-\gamma_{1}+2 \gamma_{2} x_{0}}, \\
\vartheta_{1}=\frac{\gamma_{1}^{2}}{4 \gamma_{2} \beta_{1}}, \vartheta_{2}=\frac{\beta_{1}(\varphi+1)}{2 \gamma_{2} \varphi^{2}}, \zeta(t)=\frac{1+\varphi}{1+\varphi \exp \left(-\beta_{1} t\right)}, \\
\wp(t)=\frac{\varphi\left(1-\exp \left(-\beta_{1} t\right)\right)\left(1+(1+\varphi)\left(1+\varphi \exp \left(-\beta_{1} t\right)\right)\right)}{(1+\varphi)\left(1+\varphi \exp \left(-\beta_{1} t\right)\right)}
\end{gathered}
$$

and

$$
x^{\prime}(t)=\frac{\beta_{1}^{2} \varphi \exp \left(-\beta_{1} t\right)}{2 \gamma_{2}\left(1+\varphi \exp \left(-\beta_{1} t\right)\right)^{2}} .
$$

The optimal solutions $(3.14)-(3.16)$ are feasible, provided that $\theta(t) \in(0,1)$.

On the other hand, if the total population is fixed, say $N$, then

$$
\frac{d x(t)}{d t}+\frac{d y(t)}{d t}=0
$$

so that $\beta_{1} y(t)=-\left(\gamma_{1} x(t)-\gamma_{2} x^{2}(t)\right)$. In this case, equation (3.4) becomes

$$
H=\theta(t) x(t)+\lambda(t)\left(\gamma_{1} x(t)-\gamma_{2} x^{2}(t)-\theta(t) x(t)\right),
$$

where $\lambda(t)=\lambda_{1}(t)-\lambda_{2}(t)$. The influence equations for the state variable $x(t)$ is

$$
\frac{d \lambda(t)}{d t}=-\left(\theta(t)+\lambda(t)\left(\gamma_{1}-2 \gamma_{2} x(t)-\theta(t)\right)\right) .
$$

The Lagrangian function, $L$, for the Hamiltonian subject to the control bounds, $0 \leq \theta(t)<1$, would be 


$$
L=\theta(t) x(t)+\lambda(t)\left(\gamma_{1} x(t)-\gamma_{2} x^{2}(t)-\theta(t) x(t)\right)+w_{1} \theta(t)+w_{2}(1-\theta(t)),
$$

where $w_{1}$ and $w_{2}$ are the Lagrangian multipliers when the total population is fixed. With $\theta(t)=0$, we obtain the same result as in equation (3.11). For $0<\theta(t)<1$ and $x(t) \neq 0$, we obtain $\lambda(t)=1$,

$$
\begin{gathered}
x(t)=\frac{\gamma_{1}}{2 \gamma_{2}}, \\
y(t)=N-\frac{\gamma_{1}}{2 \gamma_{2}},
\end{gathered}
$$

and

$$
\theta(t)=\frac{\gamma_{1}}{2}
$$

\subsection{Estimation of the Parameters}

In most cases, data on population are available at discrete periods, so that the discretetime model may be used to approximate the continuous-time process. Suppose historical data are available for $t=1,2, \cdots, \eta$. Then we estimate the parameters of the model by setting $\theta(t)=0$ and applying the method of least squares. By so doing, we use the difference equation

$$
x_{t}-x_{t-1}=\gamma_{1} x_{t-1}-\gamma_{2} x_{t-1}^{2}+\text { error },
$$

as the discrete-time analogue of the logistic model. Thereafter, we apply the least squares method to get

$\hat{\gamma}_{1}=\left(\left[\begin{array}{ll}1 & 0\end{array}\right]\left(\left[\begin{array}{ll}\boldsymbol{X}_{-1} & \boldsymbol{X}_{-1}^{2}\end{array}\right]^{\prime}\left[\begin{array}{ll}\boldsymbol{X}_{-1} & \boldsymbol{X}_{-1}^{2}\end{array}\right]\right)^{-1}\left[\begin{array}{ll}\boldsymbol{X}_{-1} & \boldsymbol{X}_{-1}^{2}\end{array}\right]^{\prime} \boldsymbol{X}\right)-1$,

and

$$
\hat{\gamma}_{2}=\left(\left[\begin{array}{ll}
0 & -1
\end{array}\right]\left(\left[\begin{array}{ll}
\boldsymbol{X}_{-1} & \boldsymbol{X}_{-1}^{2}
\end{array}\right]^{\prime}\left[\begin{array}{ll}
\boldsymbol{X}_{-1} & \boldsymbol{X}_{-1}^{2}
\end{array}\right]\right)^{-1}\left[\begin{array}{ll}
\boldsymbol{X}_{-1} & \boldsymbol{X}_{-1}^{2}
\end{array}\right]^{\prime} \boldsymbol{X}\right),
$$

where $\boldsymbol{X}$ is an $\eta \times 1$ vector of $x_{t}, \boldsymbol{X}_{-1}$ is an $\eta \times 1$ vector of the one period lagged number of non-adopters, $x_{t-1}$, and $\boldsymbol{X}_{-1}^{2}$ is an $\eta \times 1$ vector of the squares of the one period lagged number of non-adopters, $x_{t-1}^{2}$. We use the hat over the parameters to denote an estimate. Our approach towards obtaining the estimators for $\gamma_{1}$ and $\gamma_{2}$ is similar to the Solow method [31], except that the first-order derivative $d x(t) / d t$ is replaced by the first-order difference $x_{t}-x_{t-1}$ instead of the current value $x_{t}$ as in $[31]$.

Similarly, we obtain

$$
\hat{\beta}_{1}=\left[\begin{array}{ll}
0 & 1
\end{array}\right]\left(\left[\begin{array}{ll}
e & \boldsymbol{\Omega}
\end{array}\right]^{\prime}\left[\begin{array}{ll}
e & \boldsymbol{\Omega}
\end{array}\right]\right)^{-1}\left[\begin{array}{ll}
e & \boldsymbol{\Omega}
\end{array}\right]^{\prime} \boldsymbol{\Gamma},
$$

where $\boldsymbol{\Gamma}$ is an $\eta \times 1$ vector of $\ln y_{t}, \boldsymbol{\Omega}$ is an $\eta \times 1$ vector of time instants and $\boldsymbol{e}$ is an $\eta \times 1$ vector of ones. 


\section{Numerical Illustration}

The model defined by equations (3.14) - (3.16) is illustrated using values tabulated in Table 1 with the population size at the current period and the proportion of adopters given as $4.85 \times 10^{6}$ and 0.070 , respectively.

Table 1: The population size over time

\begin{tabular}{|c|ccccccc|}
\hline $\mathrm{t}($ in years) & 1 & 2 & 3 & 4 & 5 & 6 & 7 \\
\hline Population $\times 10^{6}$ & 2.00 & 2.28 & 2.65 & 3.12 & 3.45 & 4.19 & 4.33 \\
\hline Proportion of adopters & 0.043 & 0.041 & 0.038 & 0.040 & 0.045 & 0.049 & 0.045 \\
\hline
\end{tabular}

The parameters of the model are estimated using the least squares estimators in equations $(3.24)-(3.26)$ as $\hat{\gamma}_{1}=0.2893, \hat{\gamma}_{2}=5.1278 \times 10^{-8}$ and $\hat{\beta}_{1}=0.1593$, respectively. These parameter estimates as well as the model are statistically significant at the $5 \%$ level from the output of the MATLAB program (see Appendix). Using the parameter estimates, numerical simulations for the optimal sub-populations and the optimal fraction of new adopters are carried out and depicted in Figure 1.
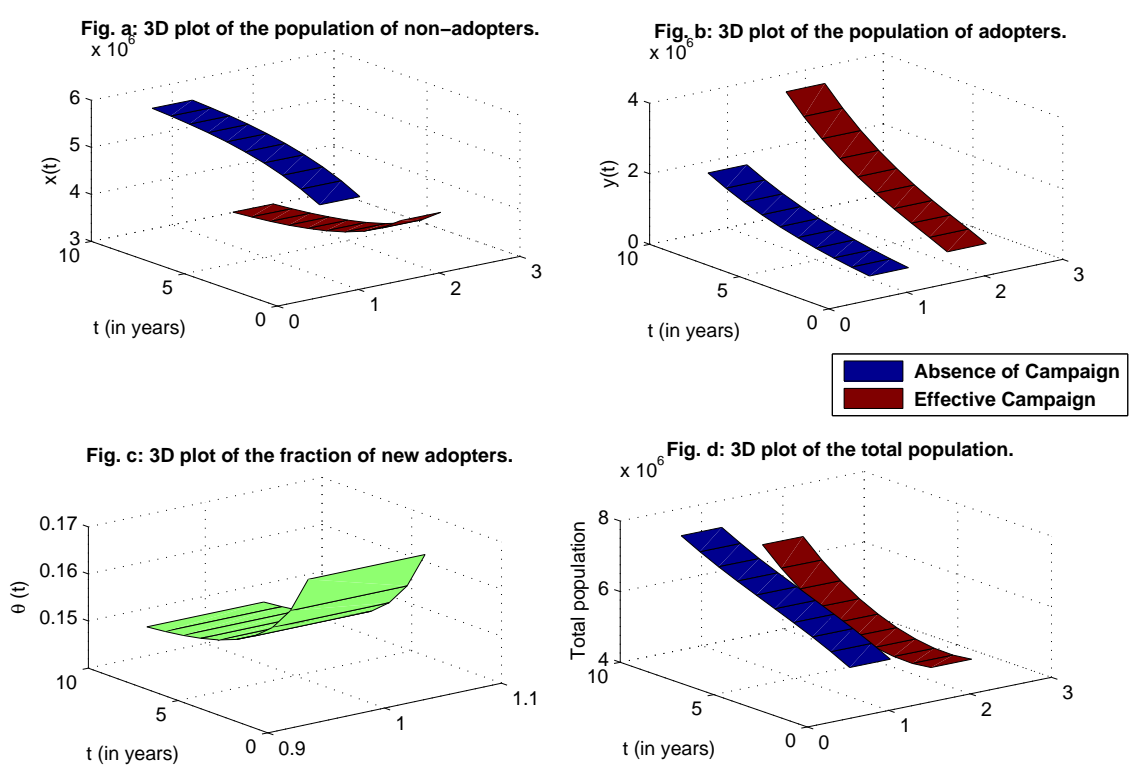

Figure 1: 3D plot of the population dynamics and the control

These simulations are performed in the MATLAB environment (see Appendix for the MATLAB source code). The simulations show that in the absence of campaign, the non-adopters and the adopters would continue to grow and the total population would rise rapidly. It is further shown that with the campaign, the non-adopters would 
reduce drastically and the adopters would increase tremendously. On the whole, the campaign is able to achieve a reduction in the total population, even though the fraction of new adopters decreases steadily. These simulations therefore suggest that the campaign on contraception is a way to improve on the use of contraceptives. Since contraception could be used as a means of population control, the model proposed in this paper is a way out of reducing population expansion.

\section{Conclusion}

This study has provided an insight into population dynamics under birth control campaign. The approach is to develop a continuous-time optimal control model to serve as an alternative to the discrete-time approach as in [23]. The fundamentals of optimal control theory and the Malthusian and logistic models of population dynamics have been used as theoretical underpinnings. The method of least squares has been employed to provide the parameter estimates. Our approach to describing population defined by two sub-populations according to the use of contraceptives is very inspiring. Nonetheless, further work may be undertaken so as to incorporate the interaction between the adopters and the non-adopters. One of the innovations of this study is to integrate population dynamics and the effect of birth control campaign in the same dynamical system, by adding the term $\theta(t) x(t)$ in the formula for both types of population dynamics, as in equations (3.1) and (3.2). This setting may be improved upon. This can be achieved by taking into consideration the time lags of the two processes as well as the delay in adopting the use of contraceptives. The time lags of the two processes, that is the population dynamics by birth and death and the transition from non-adopter to adopter, may be different. The population dynamics by birth and death takes some decades, while the transition from non-adopter to adopter happens in shorter time period, only several months or a few years. Incorporating these variables will go a long way towards refining the model as the most likely approach may involve systems of delay differential equations. Finally in the absence of subsidy, the core check and balance of driving force in the birth control campaign would be cost. In this case a well-defined cost function needs to be figured out and added as a part of the objective function.

\section{Acknowledgement}

The authors would like to thank the anonymous reviewers for their valuable comments which have greatly improved the quality of the earlier manuscript.

\section{Appendix}

clc

$\mathrm{P}=[2 ; 2.28 ; 2.65 ; 3.12 ; 3.45 ; 4.19 ; 4.33] * 10 \wedge 6$;

$\mathrm{Xlag}=($ ones $($ length $(\mathrm{P}), 1)-[0.043 ; 0.041 ; 0.038 ; 0.040 ; 0.045 ; 0.049 ; 0.045]) . * \mathrm{P}$; 


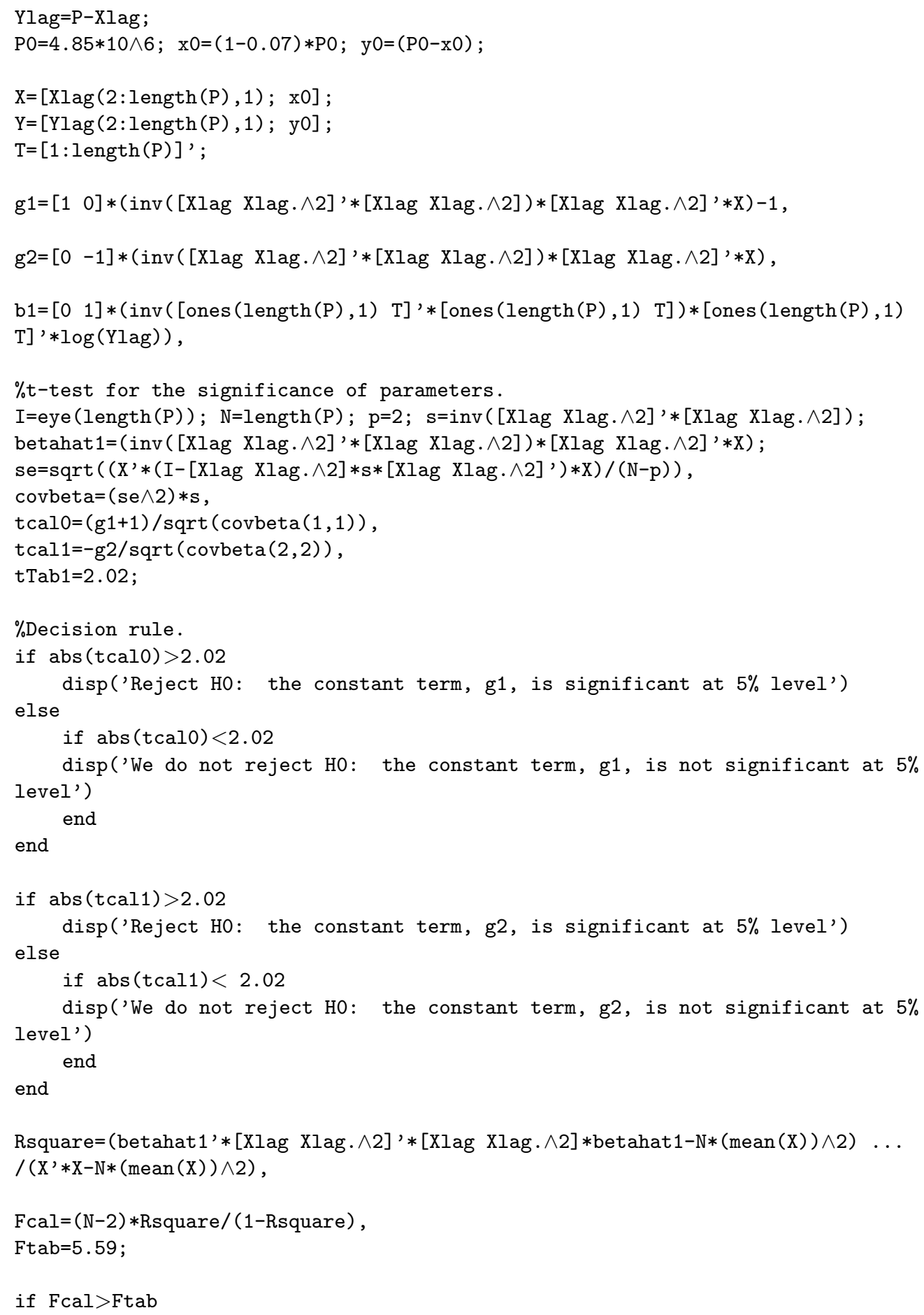




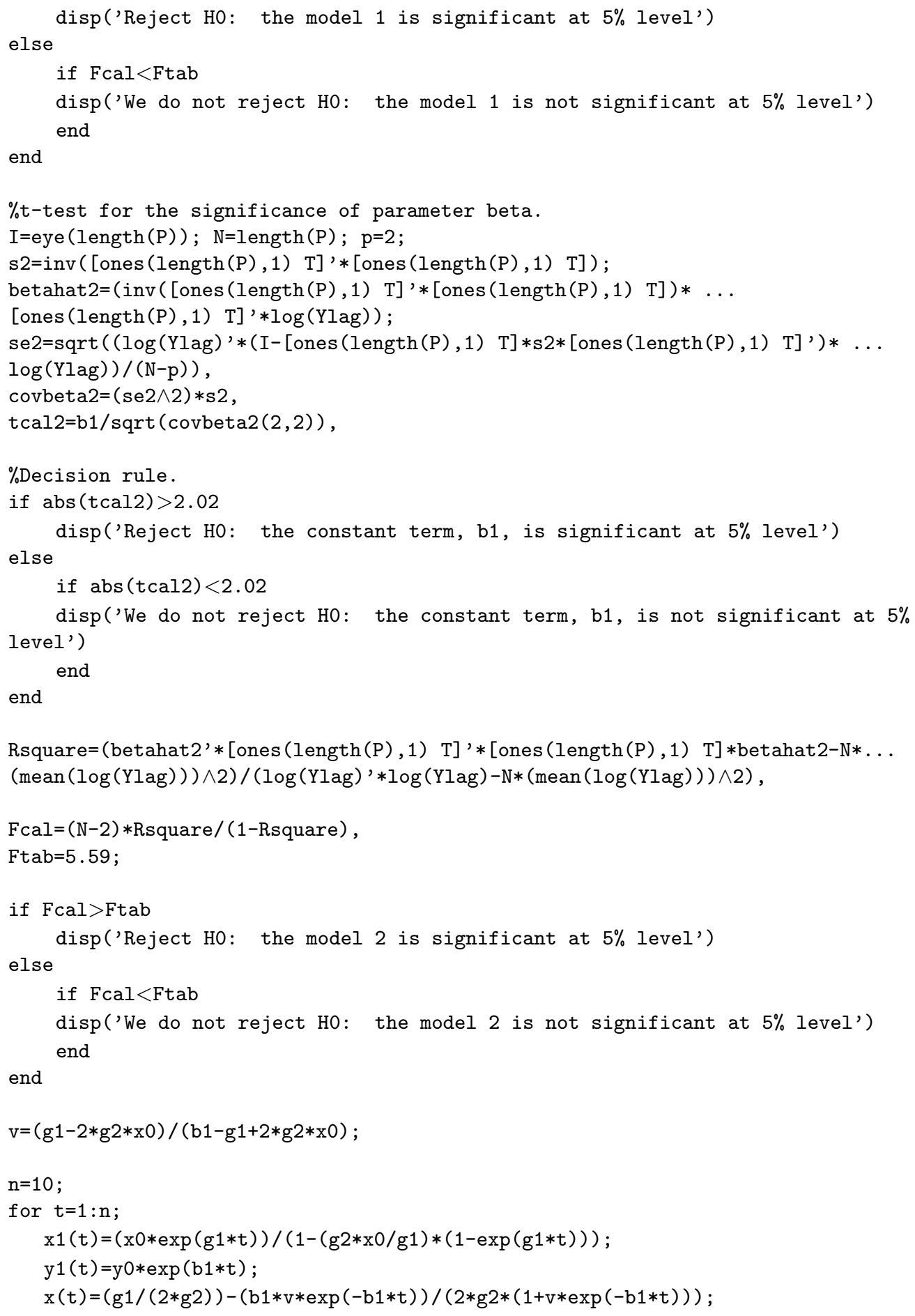




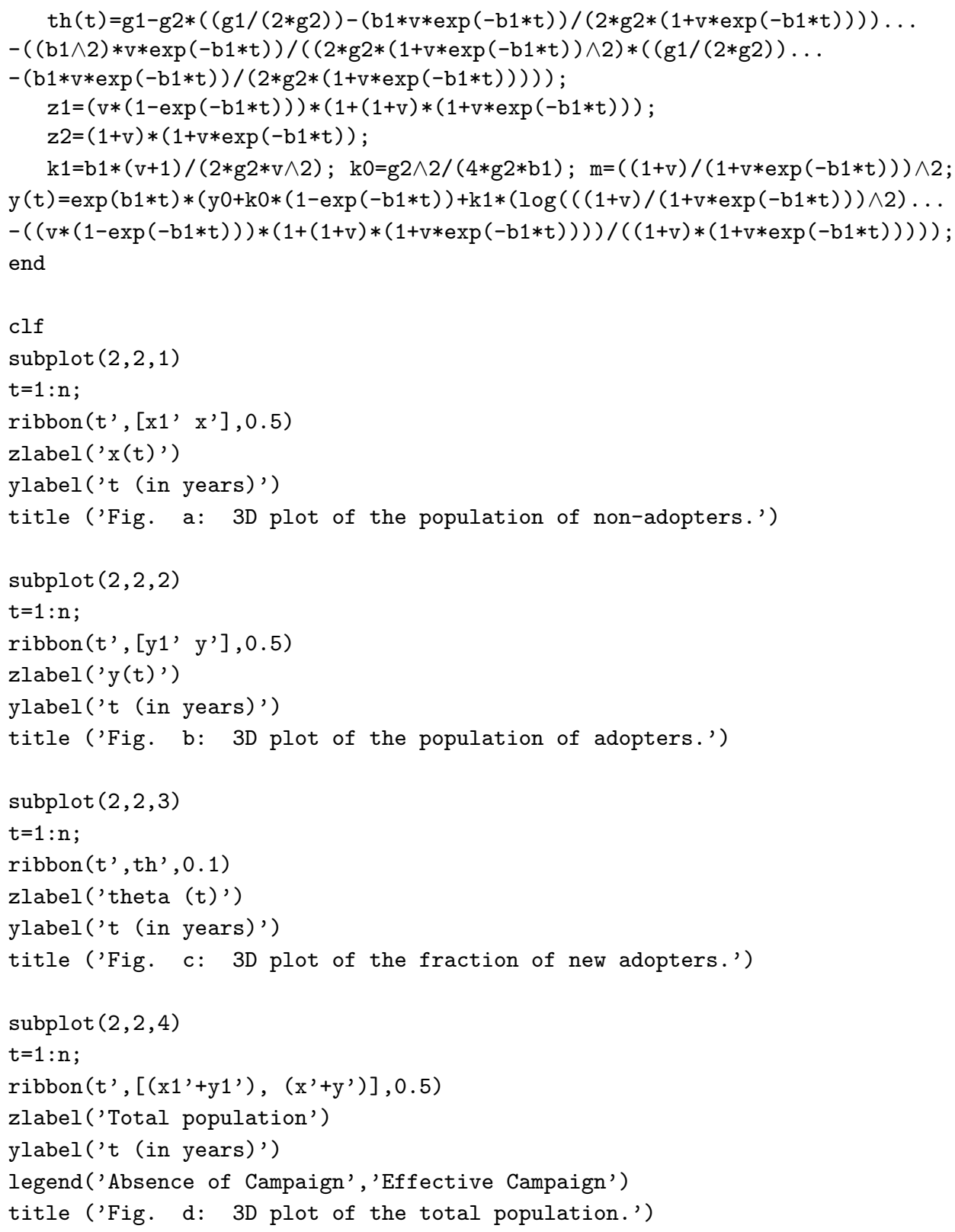




\section{References}

[1] S. Anita, V. Arnautu, V. Capasso, An Introduction to Optimal Control Problems in Life Sciences and Economics, Springer, New York, 2011.

[2] Q. Ashraf, O. Galor, Dynamics and stagnation in the Malthusian epoch, The American Economic Review 101 (5) (2011) 2003-2041.

[3] G.S. Becker, E.L. Glaeser, K.M. Murphy, Population and economic growth, The American Economic Review 89 (2) (1999) 145-149.

[4] P.A. Bourne, A.D.C. Charles, T.V. Crawford, M.D. Kerr-Campbell, C.G. Francis, N. South-Bourne, Current use of contraceptive method among women in a middle-income developing country, Open Access Journal of Contraception 1 (2010) 39-49.

[5] F. Caliendo, S. Pande, Fixed endpoint optimal control, Economic Theory, 26 (4) (2005) 1007-1012 (Accessed: 02/09/2009 from www.jstor.org).

[6] A.L. Doontchev, W.W. Hager, The Euler approximation in state constrained optimal control, Mathematics of Computation 70 (233) (2001) 173-203.

[7] J.E. Eko, K.O. Osonwa, N.C. Osuchukwu, D.A. Offiong, Prevalence of contraceptive use among women of reproductive age in Calabar metropolis, Southern Nigeria, International Journal of Humanities and Social Sciences Intervention 2 (6) (2013) 27-34.

[8] S. Enke, Leibenstein on the benefits and costs of birth control programmes, Population Studies 24 (1) (1970) 115-116.

[9] G.T. Ijaiya, U.A. Raheem, A.O. Olatinwo, M.-D.A. Ijaiya, M.A. Ijaiya, Estimating the impact of birth control on fertility rate in sub-Saharan Africa, African Journal of Reproductive Health 13 (4) (2009) 137-146 (Accessed: 22/04/2014 from www.jstor.org).

[10] A.L. Jensen, Comparison of logistic equations for population growth, Biometrics 31 (4) (1995) 853-862 (Accessed: 15/05/2014 from www.jstor.org).

[11] Y. Kwon, C.-K. Cho, Second-order accurate difference methods for a one-sex model of population dynamics, SIAM Journal on Numerical Analysis 30 (5) (1993) 1385-1399.

[12] R. Lande, S. Engen, B.-E. Saether, Optimal harvesting of fluctuating populations with a risk of extinction, The American Naturalist 145 (5) (1995) 728-745 (Accessed: 15/05/2014 from www.jstor.org).

[13] D. Leach, Re-evaluation of the logistic curve for human populations, Journal of the Royal Statistical Society, Series A (General) 144 (1) (1981) 94-103 (Accessed: 05/05/2014 from www.jstor.org). 
[14] M.L. Lee, D. Loschky, Malthusian population oscillations, The Economic Journal 97 (387) (1987) 727-739.

[15] Y. Li, Y. Kuang, Periodic solutions in periodic state-dependent delay equations and population models, Proceedings of the American Mathematical Society 130 (5) (2001) 1345-1353 (Accessed: 15/05/2014 from www.jstor.org).

[16] A.J. Lotka, Some recent results in population analysis, Journal of the American Statistical Association 33 (201) (1938) 164-178 (Accessed: 15/05/2014 from www.jstor.org).

[17] N. Meade, A modified logistic model applied to human populations, Journal of the Royal Statistical Society, Series A (Statistics in Society) 151 (3) (1988) 491-498.

[18] R.E. Mickens, Exact solutions to a population model: the logistic equation with advection, SIAM Review 30 (4) (1988) 629-633 (Accessed: 15/05/2014 from www.jstor.org).

[19] J. Mokyr, Malthusian models and Irish history, The Journal of Economic History 40 (1) (1980) 159-166.

[20] R.H. Norden, On the distribution of the time to extinction in the stochastic logistic population model, Advances in Applied Probability 14 (4) (1982) 687-708.

[21] I. Nwachukwu, O.O. Obasi, Use of modern birth control methods among rural communities in Imo State, Nigeria, African Journal of Reproductive Health 12 (1) (2008) 101-108.

[22] F.R. Oliver, Notes on the logistic curve for human populations, Journal of the Royal Statistical Society, Series A (General) 145 (3) (1982) 359-363 (Accessed: 05/05/2014 from www.jstor.org).

[23] R. Parker, Minimizing cost to maintain a steady-state growth rate in a population, Operations Research 25 (2) (1977) 326-329.

[24] R. Pearl, L.J. Reed, A further note on the mathematical theory of population growth, Proceedings of the National Academy of Sciences of the United States of America 8 (12) (1922) 365-368 (Accessed: 15/05/2014 from www.jstor.org).

[25] Prajneshu, Time-dependent solution of the logistic model for population growth in random environment, Journal of Applied Probability 17 (4) (1980) 1083-1086.

[26] H.B. Presser, M.L.K. Hattori, S. Parashar, S. Raley, Z. Sa, Demographic change and response: social context and the practice of birth control in six countries, Journal of Population Research 23 (2) (2006) 135-163.

[27] M.S. Ridout, D.J. Cole, B.J.T. Morgan, L.J. Byrne, M.F. Tuite, New approximations to the Malthusian parameter, Biometrics 62 (4) (2006) 1216-1223. 
[28] M.R. Rosenzweig, K.I. Wolpin, Evaluating the effects of optimally distributed public programs: child health and family planning interventions, The American Economic Review 76 (3) (1986) 470-482 (Accessed: 22/04/2014 from www.jstor.org).

[29] M.C. Runge, F.A. Johnson, The importance of functional form in optimal control solutions of problems in population dynamics, Ecology 83 (5) (2002) 1357-1371 (Accessed: 15/05/2014 from www.jstor.org).

[30] R.M. Schacht, Two models of population growth, American Anthropologist, New Series 82 (4) (1980) 782-798.

[31] A.R. Solow, On fitting a population model in the presence of observation error, Ecology 79 (4) (1998) 1463-1466 (Accessed: 15/05/2014 from www.jstor.org).

[32] T.J. Trussel, Cost versus effectiveness of different birth control methods, Population Studies 28 (1) (1974) 85-106.

[33] S.L. Tucker, S.O. Zimmerman, A nonlinear model of population dynamics containing an arbitrary number of continuous structure variables, SIAM Journal on Applied Mathematics 48 (3) (1988) 549-591.

[34] P.J. Wangersky, Lotka-Volterra population models, Review of Ecology and Systematics 9 (1978) 189-218 (Accessed: 15/05/2014 from www.jstor.org).

[35] S.A. Weinstein, G. Goebel, The relationship between contraceptive sex role stereotyping and attitudes toward male contraception among males, Journal of Sex Research 15 (3) (1979) 235-242.

[36] M.K. Welch, Not women's rights: birth control as poverty control in Arkansas, The Arkansas Historical Quarterly 69 (3) (2010) 220-244.

[37] P. Whittle, J. Horwood, Population extinction and optimal resource management, Philosophical Transactions: Biological Sciences 350 (1332) (1995) 179-188 (Accessed: 05/05/2014 from www.jstor.org).

\section{DOI: $10.7862 / \mathrm{rf} .2018 .6$}

Virtue U. Ekhosuehi

email: virtue.ekhosuehi@uniben.edu

ORCID: 0000-0002-7796-1657

Department of Mathematics

University of Benin

Benin City, Edo State

NIGERIA 
Fidelis O. Chete

email: odichet@yahoo.com

ORCID: 0000-0001-8445-2570

Department of Computer Science

University of Benin

Benin City, Edo State

NIGERIA

Received 05.10.2017

Accepted 13.04.2018 